\title{
Effect of Crosslinker on Swelling and Thermodynamic Properties of Polyacrylamide Gels
}

\author{
J. BASElga, I. Hernández-Fuentes, ${ }^{*}$ R. M. Masegosa, ${ }^{* *}$ \\ and M. A. LLORENTE*** \\ Departamento de Materiales y Producción Aeroespacial, \\ E.T.S.I. Aeronaúticos, Universidad Politécnica, \\ 28040 Madrid, Spain \\ * Departamento de Quimica Fisica I, Facultad de Químicas, \\ Universidad Complutense, 28040 Madrid, Spain \\ ** Cátedra de Quimica, E.U.I.T. Aeronaúticos, \\ Universidad Politécnica, 28040 Madrid, Spain \\ *** Departamento de Quimica Fisica, Facultad de Ciencias, \\ Universidad a Distancia (UNED), \\ 28040 Madrid, Spain
}

(Received September 27, 1988)

\begin{abstract}
PAA networks have been prepared by crosslinking copolymerization of acrylamide (AA) and $N, N^{\prime}$-methylen-bis-acrylamide (BA) in water. The degree of swelling was measured in the range $v_{2}=0-0.16$ as a function of crosslinker proportion, $C(\% \mathrm{w} / \mathrm{w})$ and total concentration, $C_{\mathrm{T}}(\mathrm{g} / 100 \mathrm{ml})$. The swelling of two networks with BA contents of $3 \%$ and $10 \%$ were measured as a function of temperature in the range $10-30^{\circ} \mathrm{C}$. The interpretation of elastic and swelling data in terms of the scaling law $\left|f_{\mathrm{ph}}^{*}\right|=A v_{2}{ }^{m}$, shows a transition from a good solvent regime to a collapsed state for the polymer chains, when the crosslinker proportion is around 5 to $7 \%$. Results of elastic and swelling measurements were used to determine the $\chi$ parameter. A different dependence of $\chi$ with $v_{2}$ was found for gels with BA contents below and above $5-7 \%$. The enthalpic contribution to the interaction parameter $\chi_{H}$ was calculated from swelling experiments as a function of temperature. The results show that when the BA proportion is increased, the enthalpic interaction parameter at infinite dilution gets closer to the one of poly $(N$-methyl-acrylamide).

KEY WORDS Polyacrylamide Gels / Swelling / Collapse / Interaction

Parameter /
\end{abstract}

Polyacrylamide (PAA) gels, obtained by crosslinking copolymerization of acrylamide (AA) and $N ; N^{\prime}$-methylen-bis-acrylamide (BA), are heterogeneous and this becomes evident in any property that is measured on them ${ }^{1-4}$ (see introduction of ref 1). Experimental results are usually explained supposing a two phase structure ${ }^{5,6}$ and much effort has been done to determine the origin of this heterogeneity. The compositional shift along the copolymerization process, the non uniform distribution of crosslinker ${ }^{1,7,8}$ and the extent of cyclization reactions ${ }^{9,10}$ seem to be fundamen- tal aspects which greatly modify the structure of the gels.

Typical experiments are performed varying the total concentration of comonomers, $C_{\mathrm{T}}$ $(\mathrm{g} / 100 \mathrm{ml})$, and the crosslinker proportion, $C$ $(\% \mathrm{w} / \mathrm{w})$, and the structure and properties of the resulting gels are strongly dependent on both variables. In this paper we report on the influence of the crosslinker amount on the swelling properties of this gels, in the light of scaling and thermodynamic arguments.

Scale concepts have been proved to be appropriate tools in understanding the physical 
properties of polymer systems. ${ }^{11}$ The relationship between the elastic modulus and the equilibrium polymer concentration ${ }^{11}$ (see eq 2 ) for swollen networks allow us to estimate the excluded volume parameter ${ }^{12}$ and, therefore, crossover effects between different regimes that may occur when the crosslinker proportion is varied.

On the other hand, in the thermodynamic behavior of swollen networks the interaction parameter $\chi$ plays an important role. ${ }^{13}$ The $\chi$ parameter can be obtained from swelling experiments by using the equality between mixing and elastic contributions to the solvent chemical potential if one knows how much that elastic contribution is. Here we calculate the elastic contribution from independent stress-strain measurements on the swollen networks which were previously reported. ${ }^{4}$

This method yields $\chi$ parameter values representative of the linear polymer/solvent interaction when the crosslinks have a negligible effect. Usually this requirement is met when the average molecular weight between crosslinks, $M_{\mathrm{c}}$, is high enough (above $10^{4}$ ). In the networks studied here the crosslinker content, although $M_{\mathrm{c}}$ values ${ }^{4}$ are above $10,{ }^{4}$ may affect deeply their swelling behavior. This feature will be studied by calculating the enthalpic contribution to the interaction parameter.

\section{EXPERIMENTAL}

\section{Samples preparation}

Polyacrylamide (PAA) gels were synthesized by crosslinking copolymerization of acrylamide (AA) and $N, N^{\prime}$-methylen-bis-acrylamide (BA), both monomers from Eastman Kodak, in water at room temperature. BA was used after recrystallization from acetone. The redox pair potassium persulphate (PSA)/triethanolamine (TEA), both products from Carlo Erba, was used as a room temperature initiator system; their concentrations were $\mid$ PSA $\mid=6.57 \times$ $10^{-3} \mathrm{M}$ and $\mid$ TEA $\mid=8.51 \times 10^{-3} \mathrm{M}$.

Experiments carried out at constant tem- perature, $30^{\circ} \mathrm{C}$, were performed on previously synthesized gels ${ }^{4}$ with different compositions. They were obtained varying the total comonomer weight concentration, $C_{\mathrm{T}}(\mathrm{g} / 100 \mathrm{ml})$, and the weight fraction of crosslinker in the comonomers mixture, $C(\% \mathrm{w} / \mathrm{w})$. In a set of gels, $C_{\mathrm{T}}$ was varied from 4.99 to $18 \mathrm{~g} / 100 \mathrm{ml}$, keeping constant $C=10 \%$, and in a second set of experiments $C$ was varied from 0.663 to $14.50 \%$, while keeping constant $C_{\mathrm{T}}=6.7 \mathrm{~g} /$ $100 \mathrm{ml}$. Experiments carried out at different temperatures were performed on newly synthesized gels using exactly the same polymerization conditions. The characteristics of the

Table I. Polymerization conditions, polymer volume fraction at swelling equilibrium, $v_{2}$ and elastic modulus, $\left|f_{\mathrm{ph}}^{*}\right|$, on PAA gels

\begin{tabular}{|c|c|c|c|c|}
\hline \multirow{2}{*}{ Sample } & $C_{\mathrm{T}}$ & $C$ & \multirow{2}{*}{$v_{2}$} & \multirow{2}{*}{$\frac{\left|f_{\mathrm{ph}}^{*}\right|}{\mathrm{Nmm}^{-2}}$} \\
\hline & $\mathrm{g} / 100 \mathrm{ml}$ & $\% w / w$ & & \\
\hline \multirow[t]{2}{*}{$\mathrm{T}-1$} & 18.0 & 10.1 & 0.158 & 0.311 \\
\hline & & & 0.156 & 0.336 \\
\hline \multirow[t]{2}{*}{$\mathrm{T}-2$} & 15.0 & 10.0 & 0.128 & 0.119 \\
\hline & & & 0.126 & 0.150 \\
\hline \multirow[t]{2}{*}{$T-3$} & 11.0 & 9.5 & 0.0903 & 0.0685 \\
\hline & & & 0.0862 & 0.0723 \\
\hline \multirow[t]{2}{*}{$T-4$} & 7.67 & 10.2 & 0.0587 & 0.0217 \\
\hline & & & 0.0627 & 0.0265 \\
\hline \multirow[t]{2}{*}{$T-5$} & 6.72 & 10.1 & 0.0487 & 0.0193 \\
\hline & & & 0.0484 & 0.0170 \\
\hline \multirow[t]{2}{*}{ T- 6} & 4.99 & 10.0 & 0.0338 & 0.0074 \\
\hline & & & 0.0381 & 0.0072 \\
\hline \multirow[t]{2}{*}{ C- 1} & 6.71 & 0.663 & 0.0137 & 0.0026 \\
\hline & & & 0.0124 & 0.0022 \\
\hline C- 2 & 6.60 & 1.134 & 0.0293 & 0.0093 \\
\hline \multirow[t]{2}{*}{ C- 3} & 6.70 & 2.599 & 0.0303 & 0.0130 \\
\hline & & & 0.0313 & 0.0138 \\
\hline C- 4 & 6.69 & 3.950 & 0.0403 & 0.0192 \\
\hline \multirow[t]{2}{*}{ C- 5} & 6.71 & 5.784 & 0.0463 & 0.0165 \\
\hline & & & 0.0422 & 0.0192 \\
\hline \multirow[t]{2}{*}{ C- 6} & 6.67 & 6.951 & 0.0571 & 0.0195 \\
\hline & & & 0.0501 & 0.0190 \\
\hline C- 7 & 6.68 & 8.756 & 0.0463 & 0.0154 \\
\hline \multirow[t]{2}{*}{ C- 9} & 6.70 & 11.91 & 0.0474 & 0.0135 \\
\hline & & & 0.0494 & 0.0135 \\
\hline \multirow[t]{2}{*}{ C- 10} & 6.80 & 13.98 & 0.0460 & 0.0100 \\
\hline & & & - & 0.0108 \\
\hline C-11 & 6.67 & 14.50 & 0.0487 & 0.0103 \\
\hline
\end{tabular}


whole set of gels are given in the first three columns of Table I.

All the polymerizations were carried out for two days to ensure total conversion and subsequently the samples were extracted in water in order to remove initiator residues and the soluble fraction. Further details are given in ref 4.

\section{Swelling Equilibrium Measurements}

The extracted samples were placed in a thermostated bath for one week to attain swelling equilibrium in water at $30^{\circ} \mathrm{C}$. Samples $\mathrm{T}-5$ and C-3, differing only in the crosslinker proportion $(10 \%$ and $3 \%$ respectively), were the ones chosen to carry out experiments at different temperatures; these temperatures were $30,25,20,15$, and $10 \mathrm{C}$. The samples were allowed to swell for one week at every temperature weighing them before each change.

Equilibrium polymer volume fractions were determined weighing the samples after they were vacuum dried at $50^{\circ} \mathrm{C}$, until constant weight, and assuming additivity of volumes, by means of the expression:

$$
v_{2}=\left(1+w d_{2} / d_{\mathrm{w}}\right)^{-1}
$$

where $w$ is the mass of solvent per unit mass of dry network and $d_{\mathrm{w}}$ and $d_{2}$ are the solvent ${ }^{14}$ and polymer densities respectively. $d_{2}$ was taken to be the same that the linear PAA $^{15}$ $\left(d_{2}=1.300 \mathrm{~g} / \mathrm{ml}^{-1}\right)$. In most cases two samples of each gel were measured.

\section{Turbidimetric Measurements}

Solutions of AA, BA, and initiator, with exactly the same concentration of samples C-3 and $\mathrm{T}-5$, were let to polymerize for three days inside standard quartz cells. Turbidimetric measurements were carried out on a Shimadzu UV-240 spectrophotometer at $600 \mathrm{~nm}$, were the spectrum of the sample C-3 shows any absorption band, using the standard device for temperature control.

\section{RESULTS AND DISCUSSION}

\section{Swelling Results}

Numerical values of the polymer volume fractions at $30^{\circ} \mathrm{C}$ for the samples studied, are given in column four of Table I and they are plotted against initial polymerization conditions, $C_{\mathrm{T}}$ and $C$, in Figure 1 . Two different tendencies can be observed for the two sets of gels studied. The linear increase of $v_{2}$ with the total concentration has been reported ${ }^{4}$ to be a consequence of an increase on the crosslinking density. ${ }^{16}$ Increasing $C_{\mathrm{T}}$, the probability of formation of network defects decreases (intramolecular cyclization decreases).

The variation of $v_{2}$ with the crosslinker proportion shows an asymptotic limit; first $v_{2}$ increases with $C$ until a proportion of BA around $5-7 \%$ is reached, remaining practically constnat for higher BA contents. Similar results have been reported for this system by Cohen et al. ${ }^{17}$ and our results agree also with the measurements of Richards and Temple. ${ }^{18}$

From a phenomenological point of view, it has been reported a change in this region of

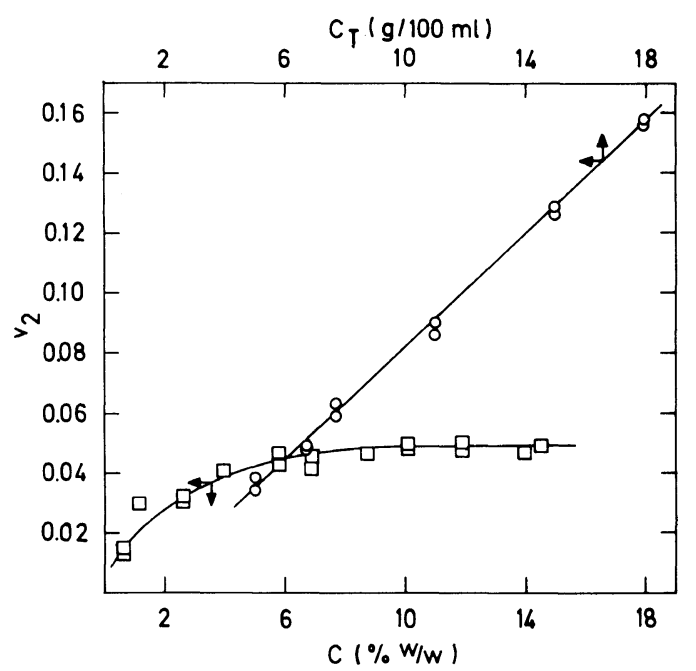

Figure 1. Polymer volume fraction at swelling equilibrium $v_{2}$, as a function of the total comonomer concentration $C_{\mathrm{T}}(\mathrm{g} / 100 \mathrm{ml}),(\mathrm{O})$, and as function of crosslinker proportion $C(\% \mathrm{w} / \mathrm{w}),(\square)$. Lines were drawn in order to show the trend of the data. 
BA concentrations on many different properties which have been measured. ${ }^{1-4}$ Coincidence on the same value of $C$ for the appearance of turbidity, ${ }^{19}$ a maximum on the elastic modulus, ${ }^{4-6}$ or a minimum on the gel point conversion, ${ }^{8}$ has been observed. The presence of high BA contents induces the formation of an increasing number of heterogeneities in PAA gels with important consequences in their final structure and topology.

Results of swelling equilibrium at different temperatures are presented in Table II. As indicated in the experimental section, two samples were studied, both with the same initial total comonomer concentration $\left(C_{\mathrm{T}}=6.7 \mathrm{~g} /\right.$ $100 \mathrm{ml}$ ), but one of them (sample $\mathrm{T}-5$ ) in the range of high BA content $(C=10.1 \%)$ while the second one (sample C-3) has a much lower amount of crosslinker monomer $(C=3 \%)$. As can be seen (Table II), a slight decrease of $v_{2}$ with temperature is observed in both gels, as expected due to the increasing solvent capacity of the water towards the PAA gels. The values

Table II. Polymer volume fraction at swelling equilibrium, $v_{2}$, interaction parameter, $\chi$, enthalpy parameter, $\chi_{\mathrm{H}}$ and enthalpy parameter at infinite dilution, $\chi_{\mathrm{H}}^{0}$, as a function of temperature for the system PAA gels/water

\begin{tabular}{lccccc}
\hline \multirow{2}{*}{ Sample } & \multicolumn{1}{c}{$T$} & & & & \\
& ${ }^{\circ} \mathrm{C}$ & $v_{2}$ & $\chi$ & $\chi_{\mathrm{H}}$ & $\chi_{\mathrm{H}}^{0}$ \\
& & & & & \\
\hline & 10 & 0.0499 & 0.495 & -0.024 & - \\
Gel T-5 & 15 & 0.0481 & 0.494 & -0.022 & - \\
& 20 & 0.0473 & 0.493 & -0.021 & - \\
& 25 & 0.0466 & 0.493 & -0.020 & 0.015 \\
& 30 & 0.0454 & 0.492 & -0.019 & - \\
& 10 & 0.0334 & 0.479 & -0.035 & - \\
Gel C-3 & 15 & 0.0326 & 0.478 & -0.032 & - \\
& 20 & 0.0312 & 0.476 & -0.027 & - \\
& 30 & 0.0302 & 0.474 & -0.024 & 0.062 \\
Linear & & & & & - \\
PAA & 25 & - & - & - & $0.08^{\mathrm{a}}$ \\
PNMA & 25 & - & - & - & $-0.06^{\mathrm{a}}$ \\
\hline
\end{tabular}

a Reference 28 . of $v_{2}$ for $\mathrm{T}-5$ gels are higher than the corresponding ones for $\mathrm{C}-3$ gels at each temperature due to its higher crosslinking density (Table I).

Turbidimetric measurements at $600 \mathrm{~nm}$ as a function of temperature, on samples C-3 and $\mathrm{T}-5$, are presented in Table III. As expected from the phase diagram of Bansil and Gupta, ${ }^{19}$ sample T-5 is opaque (high $\tau$ ) while sample C-3 is transparent (very low $\tau$ ). These features remain unchanged in the range of temperatures studied. Sample C-3 shows no substantial change of $\tau$ with temperature, although for sample T-5, turbidity slightly decreases, being more pronounced for low temperatures than for high temperatures.

\section{Elastic and Swelling Data}

Elastic data taken from ref 4 are given in column five of Table I. In Figure 2 the phantom modulus, $\left|f^{*}{ }_{\mathrm{ph}}\right|$ versus the equilibrium volume fraction is plotted, for the $\mathrm{C}$-number set of gels, in a logarithmic form in order to interpret the data in terms of the universal relationship between the elastic modulus and polymer concentration ${ }^{11}$

$$
\left|f^{*}{ }_{\mathrm{ph}}\right|=A v_{2}{ }^{m}
$$

where the factor $A$ contains the non-universal properties of the system ${ }^{12}$ while the power law exponent, $m$, is connected with the excluded volume exponent, $v$, of the free polymer chains, ${ }^{11}$ by

Table III. Turbidity $\tau$ at $600 \mathrm{~nm}$ of samples C-3 and $\mathrm{T}-5$ as a function of temperature

\begin{tabular}{|c|c|c|}
\hline$T$ & $\tau_{\mathrm{C}-3}$ & $\tau_{\mathrm{T}-5}$ \\
\hline${ }^{\circ} \mathrm{C}$ & $\mathrm{cm}^{-1}$ & $\mathrm{~cm}^{-1}$ \\
\hline 10 & 0.083 & 7.83 \\
\hline 20 & 0.078 & 7.53 \\
\hline 30 & 0.076 & 7.31 \\
\hline 40 & 0.074 & 7.12 \\
\hline 50 & 0.071 & 6.96 \\
\hline 60 & 0.069 & 6.95 \\
\hline 70 & 0.069 & 6.95 \\
\hline
\end{tabular}




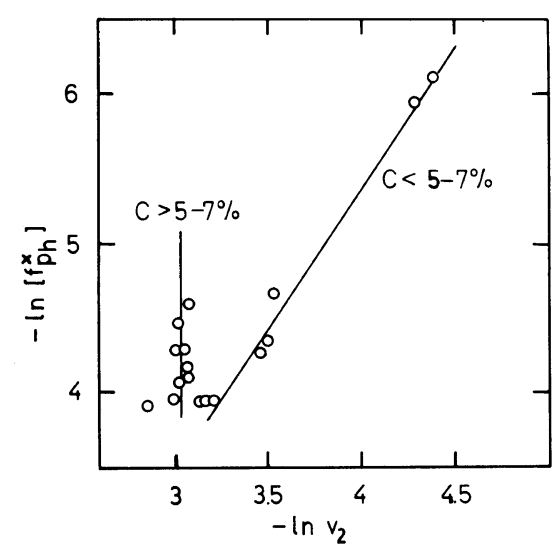

Figure 2. Logarithmic plot of the elastic modulus $\left|f_{\mathrm{ph}}^{*}\right|$ as a function of polymer volume fraction at swelling equilibrium $v_{2}$. Lines are the best fit of eq (2).

$$
m=3 v(3 v-1)^{-1}
$$

In eq $3, v$ may taken the values $3 / 5,1 / 2$, or $1 / 3$ in good, $\theta$ and poor solvents, respectively. The first two values follow readily from the mean field approach formulated by Flory ${ }^{20}$ (although for good solvents a different value has been given recently ${ }^{21}$ ), while the value $1 / 3$ results from the spherical volume occupied by a collapsed coil. Therefore the power law exponent, $m$, from eq 2 , assumes the values $2.25,3.0$, and $\infty$, respectively. However, some calculations made by Erman et al. ${ }^{23}$ show that the predicted values given above for $\theta$ and good solvents occur within a specific concentration range.

As it can be seen from Figure 2, two different behaviors can be observed for the Cnumber set of gels. The data corresponding to the samples with the lowest BA content (less than $5 \%$ ) can be fitted reasonably to a straight line (correlation coefficient 0.994 ). The slope of this line and therefore the value of $m$ is 1.88 confirming the good solvent quality of water for this system. On the other hand, for samples with BA content above $5-7 \%$, the trend of the data suggests an infinite slope which corresponds to a collapsed state of the polymer chains in the network structure. This results is in agreement with Figure 1 and elastic data given in Table I, from where it can be seen that $v_{2}$ is insensitive to $\mathrm{BA}$ proportion above $C=$ $5-7 \%$ and also, different elastic moduli for almost the same value of $v_{2}$ are obtained in this region.

A transition between a good solvent regime to a collapsed state regime seems to be induced by the increasing amount of crosslinker. The way by which such a transition is induced may be explained, taking into account also experimental observations about the development of turbidity along the polymerization process, in terms of microsyneresis ${ }^{9,23,24}$ during the formation of the gels. All these observations agree with the behavior found for other monovinyl/divinyl systems such as styrene/ divinylbenzene. ${ }^{25}$

\section{Interaction Parameter}

Let us now examine how the heterogeneities introduced in the gel structure by the increasing amount of BA affect the thermodynamics of these systems.

The chemical potential of the diluent, $\Delta \mu_{1}$, in a mixture of a collection of chain segments connected one to another as in a coherent network, may be written ${ }^{20}$

$$
\begin{aligned}
\Delta \mu_{1} & =\ln \left(1-v_{2}\right)+v_{2}+\chi v_{2}{ }^{2} \\
& +(R T)^{-1}\left(\partial \Delta A_{\mathrm{e} 1} / \partial n_{1}\right)
\end{aligned}
$$

where $\chi$ is a phenomenological coefficient, expressing deviations from purely combinatorial terms, which characterizes the interactions between solvent and polymer; $\Delta A_{\mathrm{e} 1}$ is the elastic free energy of the system, and $n_{1}$ is the number of molecules of solvent.

The last term in eq 4 represents the elastic contribution to the chemical potential of the solvent. In accordance with the theory of Flory $^{26}$ for a perfect tetrafunctional network, this last term is given by

$$
\left(\Delta \mu_{1}\right)_{\mathrm{e} 1}=\left(V_{1} / R T\right)\left|f_{\mathrm{ph}}^{*}\right| v_{2}{ }^{1 / 3}\left(1+K\left(v_{2}\right)\right)
$$

where $V_{1}$ is the molar volume of the solvent 


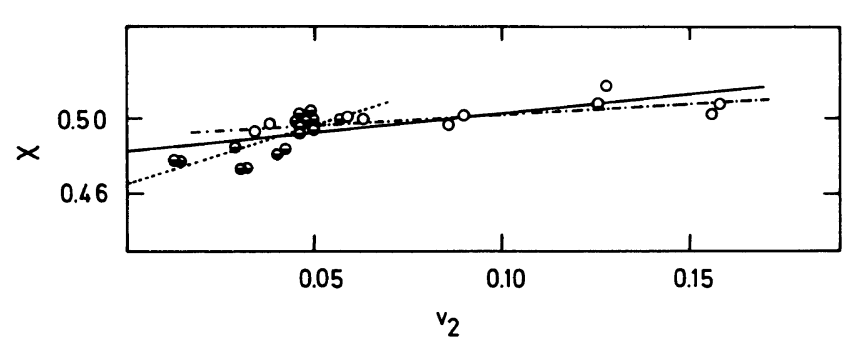

Figure 3. Interaction parameter $\chi$ as a function of polymer volume fraction $v_{2}$. $(\Theta)$ correspond to the $C$ number set of gels and $(O)$ to the $\mathrm{T}$-number set. Lines are constructed fitting experimental results to curves of the form $\chi=\chi_{1}+\chi_{2} v_{2}$ in different ranges of $v_{2}$ : (-) $0<v_{2}<0.16 ;(--\cdot-) 0<v_{2}<0.05 ;(-\cdot \cdot \cdot-)$ $0.05<v_{2} v 0.016$.

Table IV. Interaction parameter $\chi$ of the system PAA gel/water as a function of polymer volume fraction, $v_{2}$, at $30^{\circ} \mathrm{C}$ (coefficients of the equation $\left.\chi=\chi_{1}+\chi_{2} v_{2}\right)$

\begin{tabular}{ccc}
\hline $\begin{array}{c}\text { Concentration range } \\
\text { in } v_{2}\end{array}$ & $\chi_{1}$ & $\chi_{2}$ \\
\hline $0-0.16$ & 0.483 & 0.199 \\
$0-0.05$ & 0.467 & 0.610 \\
$0.05-0.16$ & 0.491 & 0.121 \\
\hline
\end{tabular}

and $K\left(v_{2}\right)$ is a function which interpolates between the affine and the phantom limits. ${ }^{27}$ This function depends on the degree of swelling and can be calculated from elastic results given in Table I and ref 4.

At swelling equilibrium the first term of eq 4 vanishes $\left(\Delta \mu_{1}=0\right)$ and the interaction parameter $\chi$, may be calculated by using eq 4 and 5 for each value of $v_{2}$.

The results obtained for the whole set of gels, at $30^{\circ} \mathrm{C}$, are presented in Figure 3. As an approximation the interaction parameter can be assumed to vary linearly with the polymer composition, $v_{2}$; therefore the data have been fitted to a straight line of the form $\chi=$ $\chi_{1}+\chi_{2} v_{2}$. Values of the constants $\chi_{1}$ and $\chi_{2}$ obtained by least square analysis are given in Table IV. The values of $\chi_{1}$ coincide with the interaction parameter at infinite dilution, $\chi^{0}$, for the system linear PAA/water, and it can be compared with other results reported in the literature for this same system. Day and
$\mathrm{Robb}^{28}$ found a value of $\chi_{0}=0.495$ for the system linear PAA/water, obtained by osmometric measurements. Similar studies ${ }^{29}$ gave values for $\chi^{0}$ in the range $0.487-0.495$, for different fractions of linear PAA. However these values are slightly higher than the value of $\chi^{0}=0.466$ obtained by a StockmayerFixman extrapolation. ${ }^{30}$ All these data confirm the favourable interaction between PAA and water.

On the other hand, if we examine Figure 3 in more detail, we can observe that the values of $\chi$ corresponding to lower polymer concentrations (values of $C$ below $5-7 \%$ ), have a different dependence with $v_{2}$ than the data obtained at higher polymer concentration (values of $C$ above $7 \%$ ). Fitting both sets of data separately we obtained the parameters also given in Table IV. We can see that the extrapolation of the data corresponding to the low polymer concentration region yields a value of $\chi^{0}$ lower than the one calculated for the most concentrated gels. This behavior is in agreement with the analysis of the elastic and swelling results made above. In the region of low values of $C$ (low concentration, $v_{2}$ ) the lower value of $\chi^{0}$ corresponds to the good solvent regime suggested by scaling arguments involved in eq 2; a value of $\mathrm{m}$ corresponding to a good solvent is obtained in this region whereas for more concentrated systems (higher values of $C$ ) a collapsed state is observed.

The behavior of the interaction parameters 
Table $\mathbf{V}$. Interaction parameter $\chi$ of the system PAA gel/water as a function of temperature $(K)$ and polymer volume fraction, $v_{2}$ (coefficients $a, b, c$, and $d$ of eq 6)

\begin{tabular}{ccrccc}
\hline \multirow{2}{*}{ Sample } & $a$ & \multicolumn{2}{c}{$b$} & & $d$ \\
\cline { 3 - 5 } & & $\mathrm{deg}^{-1}$ & & & $\mathrm{deg}^{-1}$ \\
\hline T-5 & 0.4253 & 4.5447 & 1.8798 & -226.26 \\
C-3 & 0.3503 & 18.5527 & 4.8874 & -849.06 \\
\hline
\end{tabular}

as a function of temperature has been studied on gels T-5 and C-3. As mentioned above these two samples differ in the percentage of BA monomer and they fall in different regions of the phase diagram ${ }^{4.19}$ (see Table I). The results obtained are given in Table II. In the range of temperatures investigated, the variation of $\chi$ with temperature is very small in both samples. The results have been fitted by an expression of the form ${ }^{31}$

$$
\chi=(a+b / T)+(c+d / T) v_{2}
$$

and the corresponding coefficients $a, b, c$, and $d$ are given in Table $\mathrm{V}$.

The enthalpic, $\chi_{\mathrm{H}}$, and entropic, $\chi_{\mathrm{s}}$ contributions to the interaction parameter can be calculated from its dependence with temperature ${ }^{20}$ by the expressions

$$
\begin{aligned}
& \chi_{\mathrm{H}}=-T(\partial \chi / \partial T) v_{2} \\
& \chi_{\mathrm{S}}=\chi-\chi_{\mathrm{H}}
\end{aligned}
$$

By derivation of eq 6 , values of $\chi_{H}$ for both gels have been calculated using the coefficients given in Table IV. The results are also given in Table II. As we can see, in the range of polymer concentrations covered, the values of $\chi_{\mathrm{H}}$ are negative in both cases. A negative enthalpic contribution to the interaction parameter indicates the existence of favourable polymer-solvent specific interactions.

The values calculated here for $\chi_{H}$ can be compared with the ones reported by Day and Robb $^{28}$ obtained from heats of dilution measurements, at $25^{\circ} \mathrm{C}$, on linear PAA and substituted polyacrylamides (poly $(N$-methyl acrylamide) (PNMA) and poly( $N, N$-dimethyl acrylamide) (PDMA)) in water. These authors obtain positive values for the enthalpic contribution for the system linear PAA/water. Data were obtained in the range of polymer concentrations $v_{2}=0-0.1$, and fall in the interval $\chi_{\mathrm{H}}=0.08-0.12$. The fact that in the same composition range, we obtain negative values of $\chi_{H}$ may be explained by taking into account that our gels are not constituted exclusively by pure PAA chains since the crosslinking monomer is present. Therefore our gels should be considered as copolymeric systems, and the crosslinker (BA) as a monosubstituted acrylamide monomer. Thus, the values of $\chi_{\mathrm{H}}$ obtained in these gels for all polymer concentrations, fall between the ones reported by Day and Robb in the range $v_{2}=0-0.1$, for the two systems linear PAA/water and PNMA/water $\left(\chi_{\mathrm{H}}(\mathrm{PNMA})=-0.06\right.$ to -0.015$)$.

On the other hand, by comparing the values at infinite dilution, $\chi_{\mathbf{H}^{\prime}}^{0}$ (see Table II), we can observe that in our case $\chi_{H}^{0}$ is positive for the two gels studied and its value fall also between the values corresponding to the system linear PAA/water and PNMA/water. Moreover, the two gels examined show enthalpic contributions to the interaction parameter rather different; sample T-5 (high BA content) has a value of $\chi_{\mathrm{H}}^{0}$ close to the one of the system PNMA/water and sensibly lower than the value of the gel $\mathrm{C}-3$ which is closer to the system PAA/water.

\section{CONCLUSIONS}

Elastic and swelling equilibrium measurements carried out on PAA gels, obtained with different percentages of the crosslinking monomer, show a transition between a good solvent regime, corresponding to $\mathrm{BA}$ concentrations below $5-7 \%$, and a collapsed state at higher BA concentrations.

Values of the polymer-solvent interaction parameter as a function of the polymer concentration and at constant temperature, ob- 
tained from swelling equilibrium measurements, confirm the behavior shown by the elastic experiments.

The enthalpic contribution to the interaction parameter, obtained from data of $\chi$ as a function of temperature, for different polymer concentrations, indicates the existence of favourable specific interactions in the system PAA/water. The values of $\chi_{H}$ obtained for our gels, fall between the values obtained for the systems: linear PAA/water and PNMA/water. This result is justified by the presence of $\mathrm{BA}$ units which are incorporated in the gels during their synthesis.

Acknowledgments. Financial support from CAICYT (SPAIN) under grant $n^{\circ}$ PA86/0370 is gratefully acknoweldged.

\section{REFERENCES}

1. J. L. Nieto, J. Baselga, I. Hernández-Fuentes, M. A. Llorente and I. F. Piérola, Eur. Polym. J., 23, 551 (1987).

2. T.-P. Hsu and C. Cohen, Polymer, 25, 1419 (1985).

3. V. F. Janas, F. Rodriguez and C. Cohen, Macromolecules, 13, 977 (1980).

4. J. Baselga, I. Hernández-Fuentes, I. F. Piérola and M. A. Llorente, Macromolecules, 20, 3060 (1987).

5. N. Weiss, T. T. Van Vliet and A. Silberberg, $J$. Polym. Sci. Polym. Phys. Ed. (a) 19, 1505 (1981); (b) 17, 2229 (1979).

6. N. Weiss and A. Silberberg, Br. Polym. J., 9, 144 (1977).

7. J. Baselga, M. A. Llorente, J. L. Nieto, I. HernándezFuentes and I. F. Piérola, Eur. Polym. J., 24, 161 (1988).

8. J. Baselga, I. Hernández-Fuentes, M. A. Llorente and I. F. Piérola, in preparation.

9. K. Dusek and W. Prins, Adv. Polym. Sci., 6, 1 (1969).

10. K. Dusek, Dev. Polym., 3, 143 (1982).
11. D.-G. De Glennes, "Scaling Concepts in Polymer Physics," Cornell University Press: Ithaca, N.Y. 1980.

12. M. Zrinyi and F. Horkay, Macromolecules, 17, 2805 (1984).

13. B. Erman and P. J. Flory, Macromolecules, 19, 2342 (1986).

14. H. Wagenbreth and W. Blanke, "The density of water in SI units and in the International Practical Temperature Scale," PTB-Information, 6/71, 412 (1968).

15. W. Scholtan, Makromol. Chem., 14, 169 (1954).

16. J. Baselga, I. F. a Piérola, I. Hernández-Fuentes and M. A. Llorente, Makromol. Chem., Macromol. Symp. (1988), in press.

17. T. D. Hsu, D. S. Ma and C. Cohen, Polymer, 24, 1273 (1983).

18. E. G. Richards and C. J. Temple, Nature (Phys. Sci.), 22, 92 (1971).

19. R. Bansil and M. K. Gupta, Ferroelectrics, 30, 63 (1980).

20. Preliminary experiments on determining the variation of turbidity with temperature, in the range $0-90^{\circ} \mathrm{C}$, show no substantial change although a slight decrease was observed, higher for T-5 than for C-3.

21. P. J. Flory, "Principles of Polymer Chemistry," Cornell University Press: Ithaca, N.Y. 1953.

22. J. C. Le Guillon and J. Zinn-Justin, Phys. Rev. Lett., 39, 95 (1977).

23. I. Bahar and B. Erman, Macromolecules, 20, 1696 (1987).

24. K. Dusek, Chem. Zvesti., 25, 184 (1971).

25. K. Dusek, Polym. Prepr., ACS Div. Polym. Chem., 11, 536 (1970).

26. J. Malinsky, J. Kablan and K. Dusek, Collect. Czech. Chem., 34, 711 (1969).

27. P. J. Flory, J. Chem. Phys., 66, 5720 (1977).

28. P. J. Flory and B. Erman, Macromolecules, 15, 800 (1982).

29. J. C. Day and I. D. Robb, Polymer, 22, 1530 (1981).

30. G. S. Misra and S. N. Bhattacharya, Eur. Polym. J., 15, 125 (1979).

31. See ref. 12 inside ref. 3 .

32. M. R. Gómez-Antón, R. M. Masegosa and A. Horta, Polymer, 28, 2116 (1987). 